\title{
SEC state assignment selection: consequences on the area and reliability of fault-tolerant controllers
}

\author{
R. Rochet, R. Leveugle \\ Institut National Polytechnique de Grenoble / CSI \\ 46, Avenue Félix Viallet - 38031 Grenoble Cedex - FRANCE \\ Phone: (33) 76.57.46.86 Fax: (33) 76.50.34.21 \\ E-mail: Raphael.Rochet@imag.fr
}

\begin{abstract}
This paper addresses the synthesis of fault-tolerant controller architectures based on the use of Single Error Correcting codes. The impact of two state assignment procedures, implemented in a specific synthesis tool (ASYL-SdF), is studied in terms of area and reliability. The first procedure uses a specific unseparable SEC code, while the second is based on the use of the Hamming code. This second state assignment often gives better results in terms of area and dependability, but the first state assignment remains more interesting for small controllers.
\end{abstract}

\section{Keywords}

State assignment, Single Error Correcting codes, synthesis efficiency, area, dependability

\section{INTRODUCTION}

When integrated circuits are dedicated to critical applications, it is necessary to provide faulttolerance capabilities in the major circuit blocks, in particular in the Finite State Machines (FSMs) which are implemented to control the operations performed by the circuit. For these applications, fault tolerance must often avoid any break in the service delivery. In that case, a classical solution is the Triple Modular Redundancy approach (TMR: triplication and voting). An alternative approach, initially proposed by Armstrong (1961), provides fault-tolerance in the sequencing part (next-state logic and state register) by using error correcting codes when assigning binary codes to the FSM states. This approach, studied by several authors, was expected to lead to lower hardware overheads and higher reliability than TMR (Armstrong 1961, Meyer 1971, ...). However, few practical evidences were available.

Applying this approach in the case of integrated circuits and providing the associated CAD tools was considered only recently, with the synthesis of the Single Independent Decoder (SID) 
architecture (Leveugle 1993 - FTCS). This first version of the ASYL-SdF tool used a state assignment procedure based on a specific Single Error Correcting (SEC) code, presented in (Leveugle 1993 - DAC). The analysis of the results showed that the induced area overheads were essentially due to the decoding logic (Rochet 1993). A new state assignment and synthesis flow, aiming at decreasing the size of the decoding logic, was thus developed. After a brief presentation of the basic architecture in section 2, the two state assignment procedures are presented in section 3, and the results are discussed in section 4 in terms of silicon area and dependability properties.

\section{SID ARCHITECTURE}

\subsection{Preliminaries}

A finite state machine is defined as a quintuple $(I, O, S, \delta, \omega)$ where $I, O$ and $S$ are respectively the sets of inputs, outputs and states. $\delta: I \times S \rightarrow S$ is the state transition function. $\omega: S \rightarrow O$ or $\omega:$ I x S $\rightarrow \mathrm{O}$ (Moore or Mealy-type machine) is the output function. Generally, these machines are described by their state transition graph and implemented using a register to store the current state code, and combinational logic for the next-state and output computation (Figure 1). Such an implementation without fault-tolerant capabilities is named "simplex".

The classical synthesis of a FSM involves three main phases: state assignment, Boolean equation generation, according to the binary state codes and to the $\delta$ and $\omega$ functions, and logic structure generation, including logic minimization, factorization and technology mapping. The state assignment is often performed using the minimal number of bits, which will be denoted

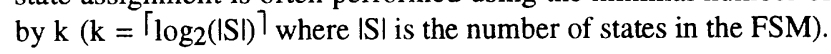

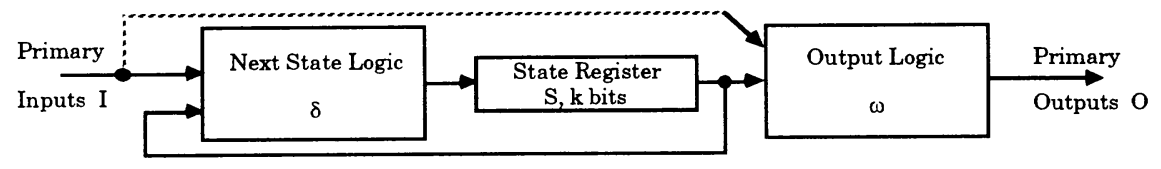

Figure 1 Simplex Finite State Machine.

\subsection{SID architecture}

The SID architecture is based on the concept presented by Armstrong (1961).With this concept, to achieve single fault tolerance in the sequencing logic (next-state logic and state register), a SEC code with a minimum Hamming distance equal to 3 must be used during the state assignment. Then, to guarantee the tolerance of a fault in the next-state logic, this logic is minimized locally and the factorization and mapping phases avoid any logic shared between two next-state functions. It can be shown that this is not really costly for this architecture, especially from the area point of view (Rochet 1993). Finally, the fault tolerance is achieved by an independent logic block connected on the outputs of the state register (Figure 2), so that an erroneous state code is corrected before it is used to compute the next-state and output functions. It must be noticed that a fault in this decoder block is not necessarily tolerated since it may directly induce errors on the outputs. 


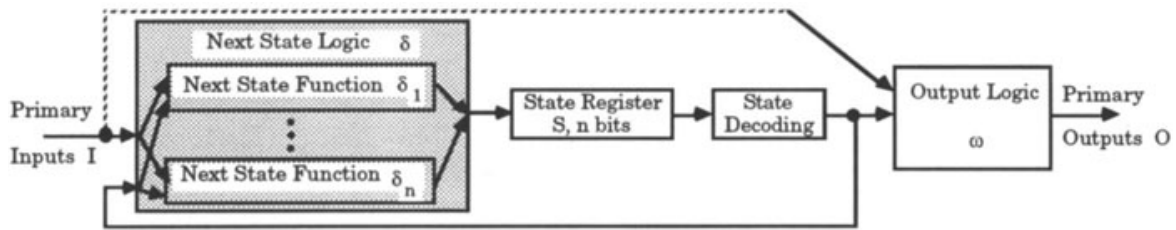

Figure 2 SID Architecture (Single Independent Decoder).

\section{STATE ASSIGNMENT PROCEDURES}

\subsection{Classical and distance 3 state assignments}

In classical optimized state assignment procedures two steps are clearly identified. The first one is the recognition, in the controller specification, of situations which will lead to minimization ; to each identified situation is associated an "adjacency constraint" on a group of states. The second one is the embedding in the hypercube, or binary code assignment, trying to satisfy as many adjacency constraints as possible (distance minimization).

A distance 3 state assignment begins with the same first step. The second step can be performed following two different approaches. The first approach, named "Global state assignment", is similar to the classical optimized assignment and tries to satisfy adjacency constraints for all state code bits, taking into account the minimum distance 3 constraint. The second approach, named "Hamming state assignment", consists in adding check bits to the optimized distance 1 state code, and then optimizes only $\mathrm{k}$ out of the $\mathrm{n}$ next-state functions.

\subsection{Global state assignment and related synthesis flow}

When considering distance 3 state assignment, the adjacency constraints cannot be handled in the same way as in the classical state assignments, which prevents us to take advantage of the existing algorithms. However, the basic principle of distance minimization can be extended to the synthesis of single fault-tolerant FSMs (Leveugle 1993 - DAC). This led to the definition of a procedure based on a specific SEC code with the following properties:

P1: The minimal distance between two code words is 3 .

P2: The total number $\mathrm{n}$ of bits necessary for any code word is the minimal known value for a SEC code, with respect to the total number of generated code words.

P3: Two successive code words are at distance 3.

P4: The sum of distances between 4 or 8 successive code words is minimum if the last word is, in the code sequence, in a position corresponding to a multiple of 4 or 8 respectively.

The embedding phase of the Global state assignment first orders the state list according to the adjacency constraints and to the distance properties of the code list, so that the distances between the codes of the states in a given adjacency constraint are minimized. Then, the $i$ th binary word of the SEC code is assigned to the $i$ th state of the ordered state list. In order to use the properties $\mathrm{P} 3$ and $\mathrm{P} 4$ at best, in a first pass, the Global state assignment searches for the constraint inclusions (inclusion of constraints is relatively easy to process by means of permutations). The result is a reduced list of constraints within which the states are partially 
ordered. The relative position of the different constraints, in the final ordered list, is then determined in a second pass according to the intersections between constraints.

For each assigned state code, the list of codes at distance 1 is built. These codes correspond to the erroneous state codes to tolerate and the decoding equations are generated so that each of these codes is mapped to the correct state code on the output of the decoding logic.

Then, the output and next-state equations are computed. In order to avoid shared logic among next-state functions, an independent local minimization, factorization and technology mapping is performed for each function. As faults are not necessarily tolerated in the output and decoding logic, each of these blocks can be globally minimized and implemented with shared logic.

As the code generated by the global state assignment procedure is not separable, the decoding block is generated with $n$ inputs and $n$ outputs $(n=k+r, r$ being the number of redundant bits). With a separable code, only $k$ outputs would be necessary for the decoder block (see section 3.3). Due to the minimization simplifications, the next-state and output equations generally use only a subset of the current state code bits to compute the next-state code and primary outputs. So a procedure is implemented to eliminate the unnecessary decoding functions. This allows us to optimize the decoding logic, but the resulting number of functions is often greater than $\mathrm{k}$.

\subsection{Hamming state assignment and related synthesis flow}

The analysis of the results obtained with the Global state assignment demonstrated that the most critical block, in terms of implementation penalties, was the decoding logic (Rochet 1993). The second state assignment procedure we implemented aims therefore at simplifying this logic, potentially at the cost of a complexity increase of the other blocks. A Hamming SEC code was selected for the state assignment. This code is known to lead to small decoder implementations. Furthermore, using this separable code, the decoder structure can be precisely controlled during the synthesis, and the minimum number $\mathrm{k}$ of outputs can be easily guaranteed.

Once the decoding logic has been optimized, it is then necessary to avoid a too large overhead in the next-state and output logic. To reach this goal, the state assignment is performed in two phases. First, a distance 1 state assignment is performed with the same optimization procedure as used for the simplex implementation, e.g. an adjacency constraint recognition followed by an optimized embedding (Saucier 1990). More optimization constraints can be satisfied during this distance 1 state assignment, compared with a global distance 3 state assignment. This allows us to achieve a better optimization of the output functions and of $\mathrm{k}$ out of the $\mathrm{n}$ next-state functions, compared to the global state assignment. Secondly, using the generator Hamming code matrix (Hamming 1950), redundant code bits are added to the words of the compact code in order to obtain the distance 3 Hamming SEC code.

With this approach, the functions related to the check bits are therefore not taken into account during the state assignment optimization. However, after state assignment, decoding equations can be generated using Hamming code properties. This leads to very small decoders, with

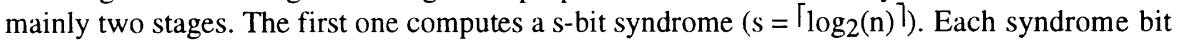
is the result of an Exclusive-OR of some state code bits. In the second stage, the syndrome value indicates which state code bit is erroneous, i.e. the bit to inverse. As only information bits are corrected, the number of outputs of the decoder block is always $\mathrm{k}$. 
After the decoder generation, the output and next-state equations are computed, minimized and factorized as described in the previous section. The $\mathrm{n}$ next-state equations compute the next-state distance 1 code plus the $\mathrm{r}$ redundant bits according to the current corrected state code (k-bit wide). The output equations compute the primary outputs according to the corrected state code.

\section{DISCUSSION OF IMPLEMENTATION RESULTS}

About 100 FSM benchmarks have been implemented using ASYL-SdF, including examples with 4 to 257 states, and 10 to 3598 transitions in the initial specification. These examples have been implemented using a CMOS $1.2 \mu$ standard cell library from VLSI Technology (vsc370) and with the area-oriented logic synthesis optimizations in ASYL. The areas after placement and routing were obtained using the COMPASS tools.

\subsection{Area Results}

Due to the Hamming code properties, the decoder block is very often smaller when the SID architecture is synthesized using the Hamming state assignment procedure rather than the Global one. This is illustrated in Figure 3, which shows the decoder size (for all implemented examples) according to the state number of the FSM and the state assignment procedure used. A very interesting characteristic of the Hamming decoding logic is to be almost not sensitive to the state number. So, even if the smallest decoders have similar sizes for the two state assignment procedures, the gain due to the Hamming assignment increases quickly with the number of states. This is explained by the regularity of the Hamming decoder structure, and the number of decoder outputs, which is guaranteed equal to $\mathrm{k}$.

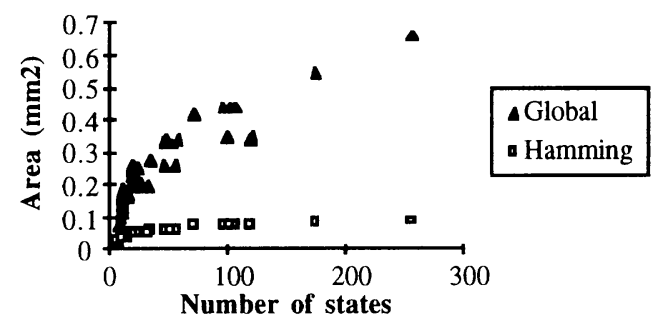

Figure 3 Area of the decoding logic for the two state assignments.

The results obtained for the next-state logic are on an average very similar for the two state assignment procedures. With the Hamming encoding, the check bit equations are not optimized, but the efficiency of the compact distance 1 state code compensates. Compared with the random distance 3 state assignment, the Global and Hamming state assignment procedures lead on an average to a sequencing logic which is $15 \%$ smaller (up to $62 \%$ smaller for Cpt 100 with the Global encoding). Results are given for some representative examples in Table 1.

Due to the optimized distance 1 code used for the $\mathrm{k}$ information bits, the output logic is very well optimized with the Hamming state assignment. So the average of the gain due to this state 
assignment procedure is greater than $20 \%$ compared with the random distance 3 encoding. The Global state assignment cannot satisfy adjacency constraints as well as the classical encoding, due to the minimum distance 3 constraint. So its average gain is only equal to $5 \%$.

Table 1 Sequencing logic area according to the state assignment procedure

\begin{tabular}{||l|c|c|c||l|l|l|l||}
\hline \hline Name & $\begin{array}{c}\text { Random } \\
(\mu \mathbf{m} 2)\end{array}$ & $\begin{array}{c}\text { Global } \\
\text { gain }\end{array}$ & $\begin{array}{c}\text { Hamming } \\
\text { gain }\end{array}$ & Name & $\begin{array}{c}\text { Random } \\
(\mu \mathbf{m} 2)\end{array}$ & $\begin{array}{c}\text { Global } \\
\text { gain }\end{array}$ & $\begin{array}{c}\text { Hamming } \\
\text { gain }\end{array}$ \\
\hline \hline cpt100 & 974804 & $62.9 \%$ & $29.3 \%$ & kirkman & 112065 & $16.4 \%$ & $21.62 \%$ \\
\hline dk17 & 89175 & $15.2 \%$ & $18.1 \%$ & s1a & 774752 & $25.3 \%$ & $27.1 \%$ \\
\hline ex7 & 143349 & $23.7 \%$ & $16.1 \%$ & sr8a-slave & 211433 & $-4.6 \%$ & $-0.6 \%$ \\
\hline imec1 & 1981778 & $43.3 \%$ & $36.3 \%$ & tav & 4309 & $-41.6 \%$ & $-49.1 \%$ \\
\hline keyb & 712141 & $22.6 \%$ & $11.3 \%$ & zeegers & 2052919 & $9.36 \%$ & $13.6 \%$ \\
\hline
\end{tabular}

In fact, even when the Global state assignment gives good results for the sequencing block, the FSM implemented with the Hamming state encoding is generally smaller, due to the output and decoding logic overheads. This is well illustrated by the ex 7 , imec 1 and keyb examples in Table 2. So the average gain of the Hamming state assignment compared with the Global one is finally $25 \%$. Actually, the Global state assignment remains interesting for few particular examples such as cpt 100 and for the small FSMs where the Hamming decoder structure does not lead to the best implementation (Figure 3).

Table 2 Gains of the Hamming state assignment compared with the Global state assignment

\begin{tabular}{||l|c|c|c|c||l|l|l|l|l||}
\hline Name & $\begin{array}{c}\text { Whole } \\
\text { logic }\end{array}$ & $\begin{array}{c}\text { Seq. } \\
\text { logic }\end{array}$ & $\begin{array}{c}\text { Out. } \\
\text { logic }\end{array}$ & $\begin{array}{c}\text { Dec. } \\
\text { logic }\end{array}$ & Name & $\begin{array}{c}\text { Whole } \\
\text { logic }\end{array}$ & $\begin{array}{c}\text { Seq. } \\
\text { logic }\end{array}$ & $\begin{array}{c}\text { Out. } \\
\text { logic }\end{array}$ & $\begin{array}{c}\text { Dec. } \\
\text { logic }\end{array}$ \\
\hline \hline cpt100 & $-5.7 \%$ & $-90.6 \%$ & $30.54 \%$ & $78.0 \%$ & kirkman & $40.9 \%$ & $6.3 \%$ & $31.5 \%$ & $71.4 \%$ \\
\hline ex7 & $25.5 \%$ & $-10.1 \%$ & $-25.1 \%$ & $68.5 \%$ & sla & $22.7 \%$ & $2.4 \%$ & N.R. & $71.4 \%$ \\
\hline dk17 & $10.0 \%$ & $3.3 \%$ & $13.9 \%$ & $6.4 \%$ & sr8a-slave & $27.0 \%$ & $3.8 \%$ & $2.1 \%$ & $78.3 \%$ \\
\hline imec1 & $31.0 \%$ & $-12.3 \%$ & $44.15 \%$ & $82.7 \%$ & tav & $3.43 \%$ & $-5.3 \%$ & $11.0 \%$ & $21.6 \%$ \\
\hline keyb & $8.45 \%$ & $-14.6 \%$ & $22.1 \%$ & $72.0 \%$ & zeegers & $17.7 \%$ & $4.7 \%$ & $13.8 \%$ & $77.9 \%$ \\
\hline
\end{tabular}

\subsection{Dependability results}

Since the FSMs are part of an integrated circuit, they are non-repairable systems and their reliability can essentially be characterized by the Mean Time to First Failure (MTFF), computed starting from the initial fault-free state, and the Mission Time (MT), representing the expected operation time for a given reliability level.

Table 3 Mission Time and MTFF (both $10^{3}$ hours) results.

\begin{tabular}{|c|c|c|c|c|c|c|c|c|c|}
\hline \multirow[t]{2}{*}{ Name } & \multicolumn{2}{|c|}{ Global } & \multicolumn{2}{|c|}{$\overline{\text { Hamming }}$} & \multirow[t]{2}{*}{ Name } & \multicolumn{2}{|c|}{ Global } & \multicolumn{2}{|c|}{ Hamming } \\
\hline & $\overline{\text { MT }}$ & $\overline{\text { MTFF }}$ & $\overline{\text { MT }}$ & MTFI & & MT & MTFF & $\overline{\mathrm{MT}}$ & MTFF \\
\hline & 462 & & 0 & & & 27192 & 111 & $\overline{48}$ & 203736 \\
\hline & 27215 & 86 & 52596 & 149 & $\log$ & 35940 & 131967 & 68243 & 208062 \\
\hline 10 & 3766 & 15696 & 5834 & 23642 & scf & 9493 & 30718 & 16127 & 45738 \\
\hline & 13711 & 46356 & 33949 & 97537 & zeegers & 5411 & 18870 & 7158 & 23621 \\
\hline
\end{tabular}


MT and MTFF have been obtained with a Markov model, using the MIL-HDBK-217F procedure to evaluate the expected failure rates (US Defense 1991) and the tool SURF-2, from LAAS-CNRS (Beounes 1993) to solve the model. The results in Table 3 illustrate the impact of the state assignment on the Mission Time (computed for a reliability equal to 0.8 ) and on the MTFF. These results show the interest to optimize the logic of the critical blocks of the SID architecture (i.e. the output and the decoder logic).

\section{CONCLUSION}

The synthesis efficiency of fault-tolerant FSMs based on SEC codes is very dependent on the efficiency of the state assignment procedure. Two state assignment procedures have been described and compared. The results show that the state assignment based on the Hamming code leads in general to a noticeable area improvement compared to the Global optimization procedure, which is however a good solution for small FSMs. In terms of reliability, the Hamming state assignment also proved its efficiency.

\section{ACKNOWLEDGEMENTS}

This work has been partially supported by the European JESSI AC8 project.

\section{REFERENCES}

Armstrong D. B., 1961, "A general method of applying error correction to synchronous digital systems", The Bell System Technical Journal, vol. 40, no. 2, March, pp. 577-593.

Beounes C. et al., 1993, "SURF-2: a program for dependability evaluation of complex hardware and software systems", 23rd FTCS, pp. 668-673.

Hamming R. W., 1950, "Error Detecting and Correcting Codes", The Bell System Technical Journal, April, pp. 147-160.

Leveugle R., 1993, "Optimized state assignment of single fault tolerant FSMs based on SEC codes", 30th DAC, pp. 14-18.

Leveugle R., Rochet R., Saucier G., Martinez L., Pitot C., 1993, "A synthesis tool for faulttolerant finite state machines", 23rd FTCS, pp. 502-511.

Meyer J. F., 1971, "Fault-tolerant sequential machines", IEEE trans. on Comp., vol. C-20, no. 10 , October, pp. 1167-1177.

Rochet R., Leveugle R., Saucier G., 1993, "Analysis and comparison of fault tolerant FSM architectures based on SEC codes", "The IEEE International Workshop on Defect and Fault Tolerance in VLSI Systems, Venice, Italy, October 27-29, 1993", F. Lombardi, M. Sami, Y. Savaria, R. Stefanelli editors, IEEE Computer Society Press, Los Alamitos, California, 1993, pp. 9-16.

Saucier G., Duff C., Poirot F., 1990, "State assignment of controllers for optimal area implementation", EDAC, pp. 547-551.

United States Department of Defense, 1991, Military Standardization Handbook: Reliability Prediction of Electronic Equipment, MIL-HDBK-217F. 